Revisión de la experiencia regulatoria en Colombia sobre las fintech y el desarrollo del sandbox regulatorio

Review of the Colombian regulatory experience on fintechs and the development of the regulatory sandbox.

Autor: Diana Pertuz Yustes

DOI: https://doi.org/10.25058/1794600X.1955 


\title{
Revisión de la experiencia regulatoria en Colombia sobre las fintech y el desarrollo del sandbox regulatorio*
}

\author{
Review of the Colombian regulatory experience on fintechs \\ and the development of the regulatory sandbox
}

\author{
Revisão da experiência regulatória na Colômbia em empresas \\ de fintech e o desenvolvimento do sanbox regulatório
}

Diana Pertuz Yustes ${ }^{a}$ dpertuzyustes@gmail.com

Fecha de recepción: 15 de junio de 2020 Fecha de revisión: 30 de junio de 2020 Fecha de aceptación: 20 de julio de 2020

\section{RESUMEN}

Es innegable que con el desarrollo tecnológico de la última década, el mundo se ha transformado de forma más acelerada. Lo es igualmente, que dicho desarrollo ha generado la creación de nuevos modelos de negocio en la industria financiera, que han cambiado de forma radical la oferta y la demanda de sus productos y servicios. Es así como las fintech se presentan como una gran oportunidad de evolución en el sector financiero tradicional, a nivel local e internacional. Éste artículo pretende realizar una descripción de los desarrollos regulatorios en Colombia frente a la aparición de las fintech; y, responder a la pregunta, de si dichos desarrollos, en conjunto con

* Artículo de reflexión elaborado como requisito parcial para optar al título de Magister en Derecho con énfasis en Derecho Privado de la Universidad del Rosario.

a. Abogada de la Universidad del Rosario (2010) y especialista en Derecho Comercial de la Universidad Javeriana (2012). Actualmente es candidata a Magister en Derecho con énfasis en derecho privado de la Universidad del Rosario.

correo: diana.pertuz@urosario.edu.co. 
el sandbox regulatorio, como mecanismo de mitigación de riesgos, son suficientes para promover de forma efectiva la innovación y modernización del sistema financiero, para una prestación más eficiente de servicios, que facilite igualmente, la inclusión financiera en Colombia.

\section{PALABRAS CLAVE}

Fintech; sandbox; regulación fintech; innovación; tecnología; sistema financiero.

\section{ABSTRACT}

It is undeniable that with the technological development of the last decade the world has been transformed in a more accelerated way. It is also undeniable that this development has generated the creation of new business models in the financial industry that have radically changed the supply and demand of financial products and services. This is how fintechs are presented as a great opportunity for evolution in the traditional financial sector at a local and international level. This article aims to describe the regulatory developments in Colombia regarding the emergence of fintechs and to answer the question of whether these developments, together with the regulatory sandbox as a risk mitigation mechanism, are sufficient to effectively promote innovation and modernization of the financial system for a more efficient provision of services that also facilitates financial inclusion in Colombia.

\section{KEYWORDS}

fintech; sandbox; fintech regulation; innovation; technology; financial system.

\section{RESUMO}

É inegável que, com o desenvolvimento tecnológico da última década, o mundo se transformou mais rapidamente. É igualmente verdade que esse desenvolvimento gerou a criação de novos modelos de negócios no setor financeiro, que mudaram radicalmente a oferta e a demanda de seus produtos e serviços. É assim que a fintech se apresenta como uma grande oportunidade de evolução no setor financeiro tradicional, local e internacional. Este artigo tenta fazer uma descrição dos desenvolvimentos regulatórios na Colômbia em face do surgimento das fintech; e, responder à pergunta, se esses desenvolvimentos, juntamente com a área restrita regulatória, como um mecanismo de mitigação de risco, são suficientes para promover efetivamente a inovação e modernização do sistema financeiro, para uma prestação de serviços mais eficiente, o que também facilita a inclusão financeira na Colômbia..

\section{PALAVRAS-CHAVE}

Fintech; sanbox; Regulamentação Fintech; inovação; tecnologia; Sistema financeiro..

\section{INTRODUCCIÓN}

Con la crisis financiera del 2008 que provocó el colapso de los mercados a nivel mundial $y$, la desconfianza de los consumidores frente al sistema financiero tradicional, comenzaron a surgir nuevos participantes y desarrollos tecnológicos e innovadores que están cambiando de forma radical la forma en la que son prestados los servicios financieros (Gurrea \& Remolina, 2020). Nos referimos a las fintech.

Las fintech son nuevas empresas financieras basadas en plataformas tecnológicas (BID, BID Invest y Finnovista, 2018) que han cambiado el concepto de la banca tradicional y, que ofrecen soluciones que están revolucionando la manera como los consumidores están guardando, pidiendo prestado, invirtiendo, moviendo, pagando y protegiendo su dinero. La agilidad para innovar y procesos centrados en la experiencia del cliente son sus ventajas competitivas (Caparroso, 2018).

Hoy en día es imposible analizar los avances de la industria financiera sin considerar el impacto que las fintech han tenido sobre la misma, no solo por el aumento del número de actores, tanto emprendimientos que operan en el espacio Fintech como entidades financieras que se acercan para interactuar con ellos, sino que también se ha visto incrementada la diversidad de los actores, entrando a formar parte del ecosistema, actores como reguladores y supervisores de los sistemas financieros, o inversionistas de capital de riesgo locales (BIS, BID Invest y Finnovista, 2018, p. 7).

En efecto, son los reguladores financieros quienes están adoptando diferentes formas de aproximarse a esta revolución, que hoy en día se constituye en un completo ecosistema $\mathrm{y}$, 
que si bien trae consigo innovación, desarrollo tecnológico y competitividad en el sector, también impone una serie de riesgos y retos desde el punto de vista regulatorio y de supervisión que deben ser abordados cuidadosamente.

Es en el marco de las fintech, que el Gobierno Nacional colombiano está implementando el espacio controlado de prueba o sandbox regulatorio $^{1}$ como se ha denominado en inglés, (arenera por su traducción al español). Este espacio controlado de prueba ha sido presentado por el Gobierno en su Decreto Reglamentario 1234 de 2020, como una herramienta de innovación pública, que fortalece las capacidades del Estado para ajustar el marco regulatorio a las nuevas dinámicas del mercado y promover una innovación financiera segura y sostenida. Es una oportunidad de innovación, por medio de la cual las empresas que tengan nuevas líneas de negocio y que quieran incursionar con nuevos productos, servicios y canales de atención, puedan operar de forma temporal, cumpliendo con los requisitos legales.

Por lo anterior, en este escrito se desarrollará la estrategia regulatoria que ha adoptado el estado colombiano en relación con las fintech, teniendo en cuenta su acelerado crecimiento y los impactos generados en el sistema financiero. Es de gran interés conocer su posición y la evolución de esta, así como responder a la pregunta de si dichos desarrollos en conjunto con el sandbox regulatorio como mecanismo de mitigación de riesgos, son suficientes para promover la innovación, el desarrollo tecnológico y la competitividad en el sector financiero colombiano.

Este escrito se divide en cuatro partes, primera, se realizará una aproximación conceptual sobre las fintech; segunda, se desarrollará la regulación financiera en Colombia, para lo cual se realizó una revisión de la regulación vigente expedida, entre otros, por la Superintendencia Financiera de Colombia (SFC), el Ministerio de Hacienda y Crédito Público y el Congreso de la República; como tercera parte, la legislación relativa a los espacios controlados de prueba o sandbox regulatorio y las funciones que cumplen, con el fin facilitar los procesos de innovación en el

1. En adelante se utilizarán indistintamente los términos "sandbox regulatorio", "bancos de pruebas" o "espacios controlados de prueba". sector financiero. Finalmente, se expondrán las conclusiones.

\section{APROXIMACIÓN CONCEPTUAL A LAS FINTECH}

La expresión "fintech" proviene de la contracción entre los términos finanzas (finance) y tecnología (technology) y se refiere a las soluciones financieras innovadoras basadas en la tecnología. A menudo se considera hoy en día, como el nuevo matrimonio entre los servicios financieros y las tecnologías de la información (Arner, Barberis \& Buckley, 2016). Estas innovaciones incluyen, entre otras: soluciones de pago móvil para consumidores y comerciantes, préstamos del mercado en línea, herramientas de inversión y ahorro algorítmico, moneda virtual, identificación y autenticación digitales biométrica del cliente, y funciones empresariales automatizadas de oficinas intermedias $y$ administrativas, como el uso de algoritmos, big data, inteligencia artificial y análisis de enlaces ${ }^{2}$ (US National Economic Council, 2017).

Si bien el uso de la tecnología siempre ha estado ligado al sector financiero, el amplio abanico de servicios mencionados $y$, los novedosos modelos de negocio que proponen las fintech para su desarrollo, pueden dar cuenta de lo disruptiva que ha sido su aparición en la industria financiera. Un estudio realizado por el Banco Interamericano de Desarrollo (2018) ha determinado que las plataformas fintech cumplen con los tres principios fundamentales de diseño para el desarrollo de un modelo de negocio que agrega valor: primero, permiten el intercambio de información entre participantes, posibilitando la toma de decisiones basadas en datos; segundo, permiten el intercambio de bienes y servicios; y finalmente, permiten el intercambio de dinero entre sus participantes.

2. Traducción libre del inglés: “... including payments, investment management, capital raising, deposits and lending, insurance, regulatory compliance, and other activities in the financial services space. These innovations include, for example, mobile payment solutions for consumers and merchants, online marketplace lending, algorithmic savings and investment tools, virtual currency, biometric digital customer identification and authentication, and automated mid-and back-office enterprise functions, such as the use of algorithms, big data, artificial intelligence, and link analytics." 
Es la velocidad, el alcance e impacto de las nuevas tecnologías lo que ha generado una revolución en la industria financiera para hacer frente a dos de los obstáculos que han impedido un mayor progreso en la inclusión financiera: (i) las limitaciones originadas en la falta de demanda, lo cual refleja la ausencia de productos que se adecúen a las necesidades y aporten valor cada uno de los diferentes segmentos; y (ii) los altos costos operacionales frente a los bajos márgenes de utilidad que representa atender a los segmentos excluidos a través de métodos tradicionales (BID, 2017, p. 17). Es así como, las fintech se han concentrado en satisfacer $y$ adaptarse a las necesidades específicas de los consumidores a través del diseño de productos y servicios más adecuados $\mathrm{y}$ asequibles para sectores tradicionalmente desatendidos o excluidos por el sector financiero tradicional (personas naturales, trabajadores independientes, pequeñas y medianas empresas y emprendimientos en general).

Ahora bien, si se hace referencia a las cifras en materia de inversión, las fintech cobran aún más relevancia, pues desde el 2018 estas han venido aumentado de manera exponencial y a la fecha, la inversión en empresas fintech en la América Latina muestra un crecimiento del 60\% (Raventós, 2020), , lo que sin lugar a duda, ha captado la atención de inversionistas y corporaciones internacionales.

De acuerdo con el informe realizado por Latam Fintech Hub (2021), durante los últimos años las inversiones en empresas fintech de Latinoamérica han aumentado de manera considerable. El informe señala que en 2020 se cerraron 199 acuerdos y se financió un monto de US\$2.989 millones, mientras que en 2019 se registraron 82 acuerdos cerrados, con una financiación total de US\$1.986 millones. Solo en el primer trimestre de 2021 se han realizado 90 rondas en las que se recaudaron US\$1.294 millones, de los cuales US\$862 millones fueron inversiones de capital $\mathrm{y}$, US\$431 millones se registraron como financiamiento de deuda. De acuerdo con el ranking de la región, los países con mayor inversión en fintech en lo corrido del 2021 son Brasil, con un recaudo de US\$969 millones, lo que representa una participación de 74,9\%; México, con $16 \%$ del mercado e inversiones por US\$207 millones; Colombia que registró 3,7\% de la industria, con un recaudo de US\$48 millones.
Por su parte Chile con US\$41 millones, Argentina con US\$15 millones y Perú (US\$3 millones) se mantienen en el ranking regional.

Las soluciones ofrecidas por las fintech a través de diferentes líneas de negocio varían ampliamente, sin embargo, todas brindan a los consumidores mayor acceso a servicios financieros de diferente naturaleza. En América Latina las modalidades que predominan son las de financiación alternativa y de pagos, seguidas por las plataformas de gestión financiera, en las que se enmarcan las plataformas de gestión de finanzas empresariales y gestión de finanzas personales. Además, existen otros emprendimientos que han ido tomando impulso: los de empresas de tecnología para instituciones financieras, negociación de activos financieros y mercado de valores, gestión patrimonial, seguros, bancos digitales, educación financiera y puntaje crediticio (BID, 2017).

La anterior segmentación permite advertir que existen productos y servicios que presentan mayores limitaciones y deficiencias para los consumidores en el sector financiero tradicional $\mathrm{y}$, que son los que generan la exclusión de ciertos sectores de la población, y las propuestas de los emprendedores fintech se han concentrado en ellas y han logrado dar una respuesta a dichas necesidades.

\section{DESARROLLO Y CRECIMIENTO DE LAS FINTECH EN COLOMBIA}

En Colombia los avances y el crecimiento de la industria fintech son cada vez más evidentes. Hoy en día se registran 322 empresas en diferentes segmentos, lo cual sitúa al país como el tercero con mayor desarrollo en la región. De acuerdo con la nueva versión de Fintech Radar Colombia, Colombia demuestra así ser, uno de los jugadores más resistentes de Latinoamérica, tanto en tiempos de crecimiento general dentro del sector Fintech como durante la incertidumbre macroeconómica global. El país andino continúa teniendo tracción y se consolida como el tercer ecosistema de la región, con un total de 200 startups Fintech, lo que le sitúa como uno de los principales mercados de Fintech en América Latina durante otro año más, solo detrás de México y Brasil (Finnovista, 2020). 
En línea con la segmentación de la región, en el país actualmente se pueden identificar dos grupos de segmentos: los segmentos principales y los segmentos emergentes. En cuanto a los segmentos principales, el de préstamos [crédito digital], se sitúa como líder por primera vez en la oferta de servicios Fintech, con 48 startups, abarcando casi el $25 \%$ del sector. Seguido por pagos [digitales] y remesas con 43 emprendimientos, representa ya el $22 \%$ del mercado; mientras que el segmento de Tecnologías Empresariales para instituciones financieras, cuenta con 27 startups y supone un $14 \%$ del ecosistema. También destacable es el segmento de Gestión de Finanzas Empresariales con 25 soluciones Fintech y un 13\% del ecosistema (Carmona \& Asociación Colombia Fintech, 2020).

Lo anterior permite entender la razón por la cual el sector fintech colombiano se ha convertido en la vía para alcanzar avances significativos en inclusión financiera, pues con su acelerada evolución en el mercado, tanto el acceso como el uso de productos y servicios financieros en el 2020 han aumentado de manera considerable. Gracias a los avances del ecosistema fintech, un gran porcentaje de personas, tradicionalmente excluidas del sector financiero, se han visto incluidas en el mismo ${ }^{3}$. Ni qué decir de los efectos de la crisis provocada por el Covid19, la cual hizo evidente la necesidad del sector financiero tradicional de adoptar un modelo de negocio más innovador y digital, así como la necesidad de colaboración entre los bancos y las fintech para efectos de brindar un mejor servicio y atención a los consumidores financieros.

3. De acuerdo con la Asociación Colombia Fintech, dentro del top 11 de fintechs en Colombia se encuentran las siguientes: Addi: ofrece soluciones de crédito y banca en punto de venta en el país, con tan solo tener un documento de identidad y celular; Bold: ofrece una solución de servicios financieros para las PYMEs y emprendimientos colombianos. Cuentan con un datáfono Bold que es un terminal de pagos móvil y un link de pago en línea que recibe tarjetas de crédito y débito; Mesfix: ofrece servicios de crowdfactoring y mercado en línea de facturas por cobrar; Moviired: ofrece servicios de transacciones electrónicas; Payu: ofrece una herramienta de pagos a comerciantes en línea; $R 5$ ofrece una plataforma de préstamos y seguros en línea a una sector de la población que no cuenta con historial crediticio: Sempli: ofrece servicios de préstamos en línea 100\% digitales para micro y pequeñas empresas; Simetrik: ofrece un software para tener visibilidad y control de los movimientos financieros de las compañías; Tpaga: app móvil para realizar pagos, retirar y comprar: Tranqui: ofrece asesoría digital para la administración de las finanzas personales; Zinobe: ofrece productos de crédito a personas naturales o pequeñas empresas; Rappi Pay: plataforma de pagos electrónicos.
Según el Reporte de Inclusión Financiera emitido por la Banca de Oportunidades para el primer semestre de 2020, el 83,6\% de la población adulta del país tenía al menos un producto de depósito en el sistema financiero, es decir 30,1 millones de personas. Un semestre atrás, este indicador equivalió a 79,3\% lo que refleja un crecimiento de más de 4,2 pp. No obstante, los productos digitales fueron los que tuvieron mayor crecimiento durante el primer semestre del año. En efecto, el número de adultos con depósitos electrónicos aumentó en 576 mil durante el primer trimestre y cerca de 3,6 millones para el segundo. De manera similar, el crecimiento del porcentaje de adultos con productos de depósito activos estuvo representado, especialmente por los productos digitales. Durante el primer semestre, el crecimiento de adultos con productos de depósitos electrónicos activos fue de más de 5,2 millones y de las cuentas de ahorro de tramite simplificado de más de 2,3 millones (Banca de Oportunidades, 2020.

Teniendo en cuenta lo anterior es posible afirmar que:

[L]as soluciones FinTech tienen un impacto positivo en el proceso de la inclusión financiera debido a cuatro componentes clave: a) Permiten procesos de identificación y verificación de clientes de manera rápida, a bajo costo y sin restricciones de tiempo y lugar. b) Abordan los problemas de distribución y servicio a través de puntos de acceso físico de bajo costo, utilizando los teléfonos móviles como herramientas de gestión del servicio. c) Mejoran significativamente el acceso al crédito mediante el uso de fuentes alternativas de datos, como transacciones de pago y datos de telecomunicaciones, los cuales mejoran el perfil del cliente al momento de la evaluación del riesgo de crédito. d) Los ahorros se pueden movilizar digitalmente a través de canales alternativos de menor costo con diseños de productos más convenientes, como billeteras móviles conectadas a cuentas de ahorro (Restrepo, 2020, p. 21).

\section{DESAFÍOS DE LAS FINTECH EN COLOMBIA}

Se ha enfatizado en las ventajas que ofrecen los desarrollos tecnológicos liderados por las fintech para los consumidores financieros, especialmente 
en lo que respecta a la inclusión financiera, las soluciones creativas e innovadoras, servicios más personalizados, así como el aporte de mayor eficiencia y reducción de costos y tiempos de transacción. Sin embargo, y a pesar de las grandes ventajas, esta nueva realidad digital también trae consigo una serie de retos y riesgos que han alertado a los reguladores y supervisores para normar de forma específica la actividad financiera desarrollada en plataformas tecnológicas para su mitigación.

La gestión del riesgo en el sector financiero tradicional se ha concentrado en mitigar los riesgos de crédito, de mercado $y$, de liquidez, entre otros, y si bien la regulación al respecto se ha ido transformando, principalmente después de la crisis de 2008, lo cierto es que las reformas de la regulación financiera promulgadas desde la crisis se han basado en una idea anticuada de cómo son y cómo se prestan los servicios financieros. La regulación no ha tenido en cuenta el auge de las empresas de tecnología financiera (o "fintech") y los cambios fundamentales que han introducido en diversos frentes, desde la forma en que funciona la banca y la manera en que se obtiene el capital, hasta la propia forma del dinero (Magnuson, 2018).

Está claro que el modelo de negocio de las fintech debe atender otro tipo de riesgos asociados a:

- El adecuado conocimiento del cliente: debido a la velocidad en la vinculación de los consumidores, pueden existir debilidades en cuanto a la veracidad de la información suministrada $\mathrm{y} / \mathrm{o}$ recopilada para lograr la plena identificación del posible cliente y su caracterización en relación con su actividad económica, su transaccionalidad y su perfil dentro del segmento de mercado al cual pertenece. Por ello, es necesario que las entidades, sean bancos tradicionales o fintechs, fortalezcan sus procesos de conocimiento del cliente.

- Protección de datos: en la era digital los datos se han convertido en el activo más valorado, sin embargo, su uso masivo trae una amenaza intrínseca a la privacidad de la información de los clientes o potenciales clientes, lo que a su vez conlleva un riesgo reputacional para las entidades en los eventos en los que no logren proteger adecuadamente los datos de sus usuarios.
- Riesgo de modelo: se ha indicado que el modelo de negocio de las fintech es diferente al de la banca tradicional, es innovador y creativo y se basa en las nuevas tecnologías, con las cuales se ha permeado el negocio bancario desde el nivel operativo hasta el comercial. Sin embargo, estos nuevos modelos de negocio pueden generar perdidas en aquellos casos en los que se tomen decisiones erradas debido a problemas con la calidad de los datos, errores técnicos o simplemente por su inadecuado uso.

- Riesgo cibernético: es tal vez el riesgo operacional más relevante 4 . Fraudes, obtención indebida de información, vulneración de datos, ciberataques, son algunas de las amenazas a las que están expuestas las entidades. y que pueden afectar de forma grave la seguridad, la operatividad y los recursos de las entidades y de sus clientes (Asobancaria, 2019).

\section{AVANCES EN REGULACIÓN DE LA ACTIVIDAD FINTECH EN COLOMBIA}

Se ha mencionado la trascendencia actual y el acelerado desarrollo de la industria fintech en el mundo en general, y en Colombia ${ }^{5}$ en particular, teniendo en cuenta que se ubica en el tercer lugar con mayor crecimiento en América Latina.

Así mismo, que tal crecimiento de las fintech trae consigo retos regulatorios $\mathrm{y}$ de supervisión que es necesario abordar para brindar mayor claridad a los actores, sin generar

4. Dentro de las tipologías más frecuentes se encuentran: (i) software espías y malware, (ii) ingeniería social, y (iii) phishing dirigido para tener acceso a los sistemas del banco. Lo anterior se ve respaldado por el "Informe Global de Riesgos de 2019" del Foro Económico Mundial, que posiciona los ciberataques y el robo de datos dentro de los cinco principales riesgos con mayor probabilidad de suceso. Véase también: Ramírez, Javier. Retos Regulatorios de las Fintech 3.0 En busca del equilibrio entre estabilidad. 2018. "La expansión de la utilización de API y de la computación en la nube son ejemplos de posibles focos de riesgo, ya que aumentan la conectividad de los sistemas de los bancos con los de operadores que no están sujetos a regulación.

5. De acuerdo con la nueva versión de Fintech Radar Colombia los datos recopilados muestran que la mayoría de las startups provienen de la capital, Bogotá (un 65\%), seguida por Medellín (22\%) y Cali (3\%), mientras que el $10 \%$ restante se distribuye en seis ciudades diferentes. En materia de internacionalización, resulta interesante destacar que en Colombia existe un mayor porcentaje de startups que operan más allá de las fronteras nacionales (el 32\%) en comparación con mercados como México y Brasil, donde tan solo un 10\% de las startups afirman haberse expandido internacionalmente. Aun así, el número de startups colombianas que operan fuera de la región Latinoamericana todavía es reducido, solo 4 startups afirman haber ampliado su actividad fuera de la región. 
cargas regulatorias excesivas que terminen obstaculizando la innovación en el sector financiero. No obstante, la discusión sobre la conveniencia o necesidad de modificar la regulación actual o introducir regulaciones específicas se mantiene. De acuerdo con una encuesta realizada por el Banco Interamericano de Desarrollo (2018) en Colombia, el 37,5\% de los encuestados afirma que la regulación es excesiva, mientras que el $34,4 \%$ piensa, o bien que la regulación es adecuada, o que no se necesita una regulación específica.

Al respecto, el Gobierno Nacional, la Superintendencia Financiera de Colombia (SFC) y la Unidad de Regulación Financiera (URF) del Ministerio de Hacienda y Crédito Público han reconocido la necesidad de abordar los cambios e impactos de la industria fintech en el sector financiero, para mantener su estabilidad y, así mismo, promover la innovación y la inclusión financiera en un marco responsable. En tal sentido, Jorge Castaño Gutiérrez, Superintendente financiero, señaló que se han hecho avances en la preparación de un camino regulatorio comprensivo y habilitante para las fintech (SFC, 2020).

La revisión del mencionado marco regulatorio habilitante, indica que el primer avance se dio con la Ley 1735 de 2014, con la cual se crearon las Sociedades especializadas en depósitos y pagos electrónicos (Sedpe). De acuerdo con lo establecido en la citada Ley, el objeto social exclusivo de las Sedpe comprende: i) la captación de recursos a través de depósitos; ii) hacer pagos y traspasos; iii) tomar préstamos dentro y fuera del país, destinados exclusivamente a la financiación de su operación; y iv) enviar y recibir giros financieros. También están autorizadas para actuar como intermediarios del mercado cambiario de acuerdo con lo dispuesto en la Resolución Externa No. 7 de 2016 del Banco de la República.

El Ministro de Hacienda, manifestó que con esta regulación el Gobierno Nacional pretende que haya mayor competencia en la prestación de servicios financieros transaccionales, con el fin de permitir el acceso a millones de ciudadanos que antes no tenían o no usaban servicios financieros formales, en igualdad de condiciones con las entidades financieras que actualmente operan en Colombia (Asobancaria, 2015).
La Ley de las Sedpe fue posteriormente reglamentada mediante el Decreto 1491 de 2015 cuyo propósito era, de un lado, aprovechar la infraestructura tecnológica disponible y la capacidad instalada en términos de cobertura territorial de otros agentes de la economía; y, de otro, diseñar una regulación prudencial más liviana, ajustada a los riesgos que administrarán estas nuevas entidades, tal y como se indica en sus consideraciones. Al respecto, la Superintendencia Financiera emitió la Circular Externa 050 de 2016 con la cual creó un capítulo sobre las Sedpe en la Circular Básica Jurídica y modificó lo relacionado con los depósitos de dinero electrónico de personas naturales, con el fin de armonizar su contenido con lo establecido en el Decreto mencionado.

Posteriormente, el Ministerio de Hacienda y Crédito Público expidió el Decreto 1357 de 2018, mediante el cual reguló la actividad de financiación colaborativa o crowdfunding en Colombia, así como las correspondientes reglas de revelación de información, estándares operativos y de funcionamiento de la infraestructura que la actividad conlleva, los mecanismos de protección de receptores y aportantes de las financiaciones, así como las reglas de prevención de lavado de activos y administración de conflictos de interés, entre otros.

En términos generales y de acuerdo con el Decreto, el crowdfunding consiste en poner en contacto a aquellos interesados en conseguir financiación para destinarla a un proyecto productivo, con un gran volumen de aportantes interesados en invertir, a través de una plataforma electrónica que sirve de puente entre los dos. Ahora bien, el decreto autorizó dos modalidades de financiación colaborativa: la primera, mediante valores representativos de deuda; y la segunda, mediante valores representativos de capital social, lo anterior con el fin de prevenir que el crowdfunding pueda ser utilizado con fines de captación masiva de dinero o lavado de activos.

Recientemente, el Gobierno Nacional expidió los Decretos 1357 de 2018 y 1235 de 2020 con el fin de continuar promoviendo la actividad de financiación colaborativa o crowdfunding 2.0 como ha sido denominado: con la incorporación de estas nuevas reglas que trae el reciente decreto, nace lo que yo he llamado el "Crowdfunding 2.0" que se va a convertir en una opción real de 
financiación alternativa para las empresas y en una locomotora, que como complemento al sector financiero colombiano, va a jalonar la reactivación económica, manifestó Germán Jaramillo (2020), CEO de Finnova ${ }^{6}$.

En el 2018, el Ministerio de Hacienda y Crédito Público también expidió el Decreto 2443 para reglamentar lo relacionado con la inversión de los establecimientos de crédito, las sociedades de servicios financieros y las sociedades de capitalización, en el capital de sociedades de innovación y tecnología financiera, cuyo objeto social exclusivo sea el de desarrollar y/o aplicar innovaciones y tecnologías conexas al desarrollo del objeto social de las entidades financieras inversoras, sin que esto constituya una autorización para que las sociedades de innovación y tecnología financiera presten servicios financieros.

Otro de los hitos en materia de desarrollo tecnológico e innovación y, que ha promovido un mayor impulso a nivel regulatorio, corresponde al Plan Nacional de Desarrollo, Ley 1955 de 2019, en el que desde el artículo 3 se indica que el logro de los objetivos de legalidad, emprendimiento y equidad, requiere de algunas condiciones habilitantes que permitan acelerar el cambio social, a través de pactos que contienen estrategias transversales tales como el pacto por la ciencia, la tecnología y la innovación. La Ley busca fortalecer el acceso al financiamiento para el emprendimiento y las mipymes, fomentar la implementación de nuevas tecnologías e innovaciones en la industria de servicios financieros, entre otros, mediante la flexibilización del marco legal para el proceso de autorización de la actividad financiera y la habilitación de espacios de prueba (sandbox); optimizar, integrar y generar sinergias en el marco regulatorio para el desarrollo de actividades de ciencia, tecnología e innovación y profundizar la inclusión financiera digital (Asociación Colombia Fintech, 2020).

En línea con lo establecido en el Plan Nacional de Desarrollo, e incluso antes de su expedición, la Superintendencia Financiera ha venido emitiendo una serie de instrucciones a través de circulares

6. Finnova es la primera sociedad de financiación colaborativa con trámite de licencia de funcionamiento ante la Superintendencia Financiera de Colombia para operar el modelo de negocio bajo lo establecido en el decreto 1357 de 2018. externas para su cumplimiento por parte de las entidades financieras:

i) Circular Externa 007 de 2018, por la cual se imparten instrucciones relacionadas con los requerimientos mínimos para la gestión del riesgo de ciberseguridad teniendo en cuenta que el auge de la digitalización de los servicios financieros, la mayor interconectividad de los agentes y la masificación en el uso de canales electrónicos, entre otros elementos, han derivado en un incremento de la exposición a riesgos cibernéticos.

ii) Circular Externa 008 de 2018, por la cual se imparten instrucciones y se introducen los conceptos de ambiente de venta presente, ambiente de venta no presente y entidades administradoras de pasarelas de pago, y fijar los requerimientos mínimos que deben cumplir los establecimientos de crédito y los administradores de sistemas de pago de bajo valor que vinculen administradores de pasarelas de pago para la realización de operaciones en ambiente de venta no presente.

iii) Circular 005 de 2019, por la cual se imparten instrucciones relacionadas con el uso de servicios de computación en la nube para el almacenamiento, procesamiento y uso de información.

iv) Circular Externa 006 de 2019, por la cual se imparten instrucciones relacionadas con la seguridad y calidad para la realización de operaciones mediante códigos QR, con el propósito de promover alternativas para realizar pagos electrónicos, consolidar la estandarización e interoperabilidad de los sistemas de pago y continuar con el proceso de fortalecimiento de la inclusión financiera y la reducción del uso del efectivo.

v) Circular Externa 026 de 2019, por la cual se imparten instrucciones para el uso de dispositivos móviles en condiciones seguras, cómodas y económicas para el consumidor financiero.

vi) Circular Externa 029 de 2019, por la cual se modifica la Circular Básica Jurídica en materia de requerimientos mínimos de seguridad y calidad para la realización 
de operaciones y acceso e información al consumidor financiero y uso de factores biométricos.

vii) Circular Externa 027 de 2020, por la cual se imparten instrucciones relativas a la administración del riesgo de lavado de activos y de financiación del terrorismo (SARLAFT 4.0).

viii) Circular Externa 042 de 2020, por la cual se imparten instrucciones relacionadas con los depósitos de bajo monto, depósitos ordinarios y crédito de consumo de bajo monto, con el fin de incorporar las reglas relativas a los depósitos de bajo monto y depósitos ordinarios de que trata el Decreto 222 de 2020.

El 2020 fue también un año de producción regulatoria. El Decreto 1154 de 2020 referente a la circulación de la factura electrónica de venta como título valor, sobre el cual vale la pena mencionar, que si bien la factura ha sido considerada, desde antaño, un tipo de título valor, la factura electrónica obtuvo esta clasificación mercantil desde el Decreto 1074 de 2015. No obstante, es el decreto del 2020 el pionero en establecer las reglas de circulación y pago de estos títulos valores, otorgándole una especial función a la DIAN como validador de dichas facturas (Brigard Urrutia, 2020).

De otra parte, está el Decreto 1234 de 2020 por medio del cual se adiciona el Decreto 2555 de 2010, en lo relacionado con el espacio controlado de prueba para actividades de innovación financiera o más conocido internacionalmente como sandbox regulatorio.

Finalmente, el Ministerio de Hacienda y Crédito Público expidió el Decreto 1692 de 2020, mediante el cual modifica el Decreto 2555 de 2010 en lo concerniente a los sistemas de pago de bajo valor o pagos electrónicos. Esta norma incluye cinco cambios clave: la apertura de la actividad de adquirencia a nuevos jugadores, cambios en el gobierno corporativo de las entidades que administran sistemas de pagos, mayor transparencia en reglas de acceso a nuevos participantes de los sistemas de pagos, separación y definición de las distintas actividades en el ecosistema de pagos y definición de finalidad e irrevocabilidad del pago (Pérez Díaz, 2020).
De acuerdo con Colombia Fintech (2020), esta nueva norma permite profundizar la inclusión financiera, permitiendo ampliar accesos a las infraestructuras de pagos, esto admite disminuir los costos vinculados a micro pagos por sistemas digitales o electrónicos y además permite que nuevos actores participen.

De los desarrollos regulatorios descritos es posible afirmar que, en efecto, el Gobierno y las autoridades regulatorias en Colombia han dado pasos significativos en aspectos que abarcan la gestión de riesgos, el manejo de la información y la protección al consumidor. Así mismo, han querido abordar aspectos relacionados con los nuevos modelos de negocio para facilitar la innovación y han introducido cambios para atender aspectos relacionados con los avances tecnológicos.

\section{LA ACTIVIDAD GREMIAL A TRAVÉS DE LA ASOCIACIÓN COLOMBIA FINTECH}

El desarrollo regulatorio ha estado acompañado por el diálogo entre las autoridades, los emprendedores de las fintech y otros agentes internos y externos. Este dialogo se dirige a la producción de cambios en las políticas públicas de manera que, por un lado, fomenten e incentiven la actividad, y por el otro, la reglamenten (BID, 2017, p. 87).

Como resultado de ese dialogo, han aparecido asociaciones gremiales cuyo propósito es representar las opiniones y puntos de vista de la industria fintech, cada vez más numerosa, frente a los reguladores, impulsar y dar a conocer mejores prácticas y promover el conocimiento de las fintech en el público en general.

En Colombia esta asociación se llama Colombia Fintech. Constituida a finales del 2017, actualmente esta asociación agrupa más de 210 empresas incluyendo fintechs, entidades del sector financiero de gran reconocimiento, así como entidades del Gobierno Nacional. En poco tiempo se ha consolidado como el gremio representativo de la industria de tecnología e innovación financiera en Colombia, y una de las asociaciones fintech más dinámicas de América Latina. De acuerdo con su presidente, en el año 2018 la asociación tuvo un crecimiento cercano al $177 \%$ tanto en ingresos operaciones como en el número de afiliados y de miembros asociados, que son dos categorías distintas: asociados, son 
los que realizan directamente actividades fintech; y afiliados, son algunas entidades financieras que están interesadas en trabajar en temas de tecnología financiera (DataIFX, 2020).

La labor más importante del gremio es llevar la vocería del sector ante el Gobierno y entes internacionales, destacando sus retos y desafíos, aportando insumos en diferentes proyectos de ley y política pública, así como crear una propuesta de valor para asociados y afiliados que vaya más allá de lo regulatorio, facilitar la creación y conexión de vehículos de financiamiento con los asociados de acuerdo con sus diferentes etapas de crecimiento, para la atracción de inversión.

Es claro que una asociación como Colombia Fintech es de suma relevancia, pues a través de este tipo de iniciativas ha sido posible empoderar al sector como gremio en el país y fortalecer su capacidad de diálogo y de negociación con el Gobierno y el sector financiero tradicional (BID, BID Invest y Finnovista, 2018, p. 88). Al apoyar y promover ese dialogo es que se ha generado un mayor conocimiento acerca de la industria fintech, de los modelos de negocio y de las experiencias en otros países de la región.

\section{EL SANDBOX COMO MODELO DE REGULACIÓN Y SUPERVISIÓN EN LA INDUSTRIA FINANCIERA}

La palabra en inglés sandbox traduce "caja de arena”. Una traducción común del término hace referencia a una arenera o espacio lleno de arena, en donde se practican juegos infantiles. En el ámbito financiero, un sandbox es un espacio controlado por el regulador en el que se puede experimentar. La palabra no es propia de los reguladores financieros, sino que proviene del sector tecnológico donde un "sandbox" representa un ambiente de prueba aislado para monitorear un nuevo software o proceso (Gurrea, 2020, p. 158).

De la combinación de ambas ideas, el de la arenera para juegos infantiles y el del ambiente aislado de prueba, surgen los sandboxes regulatorios en el sector financiero. No son los únicos tipos de sandbox, pero sí los más utilizados en la comunidad internacional y a los que se refiere el presente artículo ${ }^{7}$. Serían una suerte

7. Para ampliar la información acerca de otros tipos de sandbox, de espacios de experimentación, que permiten a empresas innovadoras operar temporalmente, bajo ciertas reglas que limitan aspectos como el número de usuarios o el período de tiempo en que se puede ofrecer el producto. De esta forma las empresas pueden probar productos, servicios y soluciones originales, ante la atenta mirada del supervisor (Herrera \& Badillo, 2018). Desde el punto de vista de la regulación financiera, los sandboxes son un instrumento de uso relativamente reciente, pero favorablemente recibido e implementado por reguladores de diferentes países. Surgieron, precisamente, como respuesta a los retos derivados de la aparición de las fintech cuya actividad de naturaleza financiera, es considerada de interés público. Sin duda, ha sido la tendencia regulatoria a nivel internacional, pues en la actualidad, más de 50 jurisdicciones han implementado sandboxes a pesar de no existir evidencia empírica de su aporte real a la innovación financiera (Gurrea, 2020, pp. 158, 160).

En algunas regiones, el establecimiento de sandbox se encuentra en un estado avanzado,

[S]in embargo, América Latina aún no llega al nivel de Europa o Asia, donde ya existen programas colaborativos entre importantes actores de la industria como los siguientes: (i) Startupbootcamp Fintech Singapore o Startupbootcamp Insurtech London, (ii) bancos de prueba regulatorios (sandboxes) como los adoptados por los reguladores del Reino Unido (Financial Conduct Authority) y Singapur (Monetary Authority of Singapore), y (iii) fondos de capital de riesgo corporativos para Fintech como los lanzados por BBVA y Banco Santander. (BID, 2017, p. 34).

No obstante lo anterior, en América Latina el lanzamiento de sandboxes es cada vez más común. Los reguladores de México, Colombia y Brasil, hoy figuran a la cabeza de la materia. Otros, como Perú y Chile, aún no tienen definido el camino que deberán recorrer para promocionar la participación en los espacios controlados (Seminario, 2021). Los siguientes son ejemplos puntuales: i) Ley Fintech de México, primera en la

tales como sandbox de supervisión, sandbox multijurisdiccionales y sandbox para promover objetivos específicos, es útil el libro Fintech, Regtech y Legaltech: fundamentos y desafíos regulatorios, de Gurrea, A., y Remolina, N. (2020). 
región y con la cual se introdujo regulación sobre "modelos novedosos"8, una autorización temporal para las instituciones de tecnología financiera ${ }^{9}$, con posibilidad de prórroga $\mathrm{y}$, la flexibilidad del entorno aislado en el que existen ciertas excepciones regulatorias para los productos y servicios novedosos. ii) Los sandboxes de Brasil subdivididos en industrias, así: sistemas financieros y de pagos, mercado de capitales y mercado de seguros privados. iii) en Colombia existen dos modalidades: una, corresponde a la arenera que fue creada por la Superintendencia Financiera de Colombia en 2018; la segunda, corresponde al espacio controlado de prueba o sandbox regulatorio creado mediante el Decreto 1234 de 2020 por el Ministerio de Hacienda y Crédito Público, ambos son espacios controlados y supervisados.

\section{SOBRE EL DESARROLLO NORMATIVO DEL SANDBOX REGULATORIO EN COLOMBIA}

Antes de la expedición del Decreto 1234 de 2020, la Superintendencia Financiera había creado, mediante la Resolución 0331 de 2017, modificada posteriormente por la Resolución 0143 de 2020, el Grupo de Trabajo de Innovación Financiera y Tecnológica - InnovaSFC, cuyos objetivos están dirigidos a acompañar la innovación al servicio del consumidor financiero, apoyar los desarrollos innovadores que promueven la inclusión y la educación financiera, así como, el desarrollo y la innovación financiera y tecnológica, y promover la innovación sostenible.

En el anterior escenario, la Superintendencia Financiera de Colombia también determinó la importancia de promover el dinamismo digital a través de herramientas facilitadoras de innovación como el Hub, la Arenera y el Regtech. El objetivo de la primera de estas herramientas, es el de servir como punto de contacto de la SFC y los interesados en el ecosistema FinTech, apoyar, asesorar, guiar y

8. De acuerdo con la Nueva Ley DOF 09-03-2018 para regular las instituciones de tecnología financiera de México, un modelo novedoso es aquel que para la prestación de servicios financieros utilice herramientas o medio tecnológicos con modalidades distintas a las existentes en el mercado al momento en que se otorgue la autorización temporal en términos de esta Ley.

9. De acuerdo con la Nueva Ley DOF 09-03-2018 para regular las instituciones de tecnología financiera de México, ITF, [se refiere] a las instituciones de tecnología reguladas en esta Ley, las cuales son las instituciones de financiamiento colectivo y las instituciones de fondos de pago electrónico. recibir retroalimentación de entidades (vigiladas y no vigiladas) en temas relacionados con la innovación financiera y tecnológica (SFC, 2018). Como oficina de innovación, el Hub permite al regulador incrementar su conocimiento para estar en mejor capacidad de regular y supervisar, y permite a los desarrolladores solventar sus dudas sobre el mercado regulatorio aplicable a sus productos. Lo anterior, sin necesidad de tener un desarrollo regulatorio previo (Currea, 2020, p. 156).

De acuerdo con el manual de funcionamiento publicado por la Superintendencia Financiera, la Arenera constituye el marco dispuesto para la realización de pruebas de innovaciones tecnológicas y financieras en un espacio controlado y supervisado. En este espacio, las entidades innovadoras podrán poner a prueba nuevos modelos de negocios, aplicaciones, procesos o productos que tengan componentes de innovación en tecnologías, que impacten en los servicios financieros y que representen un beneficio para el consumidor financiero, faciliten la inclusión financiera, desarrollen los mercados financieros o mejoren la competencia entre entidades vigiladas (SFC, 2020). La tercera herramienta para facilitar la innovación, corresponde al Regtech; su objetivo es aprovechar los desarrollos tecnológicos para apalancar la innovación al interior de la SFC, optimizando procesos internos y reduciendo cargas operativas para el sector (SFC, 2018).

En una segunda etapa de regulación, en desarrollo de lo dispuesto en el artículo 166 del Plan Nacional de Desarrollo, el Ministerio de Hacienda optó recientemente por acoger el modelo regulatorio basado en sandbox regulatorio, mediante la expedición del Decreto 1234 de 2020 con el cual se introduce un nuevo capítulo al Decreto 2555 de 2010 (Decreto Único del sector financiero) relacionado con el espacio controlado de prueba para actividades de innovación financiera.

Dentro de las consideraciones del decreto, se indica el impulso de la agenda regulatoria por parte del Gobierno Nacional para promover la transformación y digitalización de la actividad financiera, bursátil y aseguradora, manteniendo la integridad y estabilidad del sector, la debida protección del consumidor y, evitando arbitrajes regulatorios. Lo anterior, tiene sentido si se 
tiene en cuenta lo dispuesto en el artículo 166 del Plan Nacional de Desarrollo, Ley 1995 de 2019 , con respecto a la constitución de empresas de desarrollos tecnológicos innovadores, o lo dispuesto en los artículos 5 y 83 de la Ley de Emprendimiento, Ley 2069 de 2020, sobre los mecanismos exploratorios de regulación para modelos de negocio innovadores en industrias reguladas (sandbox) y la generación de espacios de articulación que permitan desarrollar programas o iniciativas que propendan por la innovación y el fortalecimiento de los modelos de negocios en las empresas e iniciativas productivas, a través del planteamiento de la solución de retos que permita la aprobación de la tecnología y de la innovación en el sector privado.

La creación del espacio controlado de prueba a través del Decreto 1234 de 2020 constituye una respuesta regulatoria del Gobierno Nacional y una herramienta más de innovación pública, que fortalece las capacidades del Estado para ajustar el marco regulatorio a las nuevas dinámicas del mercado y promover una innovación financiera segura y sostenida. De acuerdo con el decreto, en el funcionamiento, regulación y supervisión del espacio controlado se han de tener en cuenta los siguientes objetivos:

1. Aprovechar la innovación en la prestación de servicios y productos financieros.

2. Velar por la protección y los intereses de los consumidores financieros.

3. Preservar la integridad y estabilidad del sistema financiero.

4. Prevenir los arbitrajes regulatorios.

El decreto señala que dentro de las finalidades de los desarrollos tecnológicos que se acepten para participar en el espacio controlado de prueba deberán comprender: aumentar la eficiencia en la prestación de servicios u ofrecimiento de productos financieros, resolver una problemática para los consumidores financieros, facilitar la inclusión financiera, mejorar el cumplimiento normativo y, desarrollar los mercados financieros o mejorar su competitividad.

En relación con los requisitos de ingreso, el desarrollo tecnológico innovador propuesto debe estar enmarcado en alguna de las finalidades previstas $\mathrm{y}$, estar suficientemente avanzado de forma tal que pueda ser probado inmediatamente después de ser expedido el certificado de operación temporal (en este caso, será la SFC quien, establecerá los parámetros que permitan definir el estado de avance del desarrollo tecnológico innovador que pretenda ser probado en el espacio controlado de prueba), y los servicios financieros deben ser prestados en territorio colombiano.

Una vez la SFC haya aprobado la constitución para operación se expedirá al interesado un certificado de operación temporal. Este documento autorizará a los participantes para llevar a cabo la actividad propuesta, esto sujeto a las condiciones, requisitos y requerimientos prudenciales que este contenga. Una vez se cumpla el tiempo establecido en el certificado de operación temporal y el participante no pretenda realizar la transición a la licencia de entidad financiera, bursátil o aseguradora o el ajuste a la actividad regulada, según sea el caso, éste deberá activar el plan de desmonte atendiendo las instrucciones generales que para el efecto dicte la Superintendencia Financiera de Colombia.

Gracias a la creación del sandbox regulatorio, recientemente la Superintendencia Financiera anunció el primer proyecto de prueba de transacciones cash-in (depósito de recursos) y cash-out (retiro de recursos) a través de plataformas de intercambio (exchange) de criptoactivos $^{10}$. Resulta ser una noticia positiva para los servicios financieros que le apuestan al Blockchain, y para Colombia Fintech como Asociación de Empresas Fintech de Colombia, debido a que dentro de este servicio económico digital se busca lograr mayor alcance en inclusión financiera, pagos instantáneos, bajos costos y reducción de fraudes. Incluso, la expansión de estos servicios digitales beneficia en su mayoría a los clientes de la banca, al contar con un mayor portafolio de opciones financieros a elegir (Rojas, 2020). Debe recordarse, sin embargo, que la industria fintech se desarrolla en varios segmentos, como se ha indicado (crédito digital, pagos digitales, finanzas empresariales, crowdfunding, etc.), cuyos servicios y productos de

10. Las alianzas que pasaron fueron Banco de Bogotá - Bitso Banco de Bogotá - Buda, Bancolombia - Gemini, Coltefinanciera - Obsidiam, Davivienda - Binance, Powwi - Binance, Coink Banexcoin, Movii - Panda y Movii - Bitpoint. 
los que hoy en día se benefician los consumidores, sin haber atravesado por el proceso del sandbox. Por lo cual, nuevamente es necesario cuestionarse si todos esos modelos de negocios podrían adaptarse a un modelo de concesión de licencias como el que resultaría de un sandbox exitoso.

En este punto es oportuno mencionar que no es la innovación en sí misma la que debe regularse, pues como se ha evidenciado, especialmente durante la crisis generada por el Covid-19, esta va a una velocidad superior a lo que podría ir el desarrollo regulatorio o legislativo y su prohibición o limitación no solo serían contraproducentes, sino poco probables. Entonces, si bien el decreto representa un avance para el país desde el punto de vista regulatorio, existen voces que señalan que no es suficiente y que se requiere un mayor desarrollo.

En efecto, en países como México ${ }^{11}$ se ha visto cómo los sandboxes tienen una serie de limitaciones para los emprendedores, pues no está probado el grado de supervivencia de los nuevos modelos de negocio fuera de los entornos controlados, particularmente porque una vez culminado el plazo de prueba pasan a cumplir con las cargas regulatorias y administrativas de la Ley, que pueden ser muy pesadas para algunas startups. Lo que termina desincentivando a las empresas de economía digital y fintechs. Por otro lado, las autorizaciones provisionales son insuficientes para que una nueva empresa alcance solidez (Seminario, 2021), sobre todo si tiene que absorber la carga regulatoria de una entidad vigilada.

Al final, el modelo de sandbox regulatorio pareciera querer incluir en una misma categoría a entidades de diferente naturaleza e imponer las mismas obligaciones y cargas regulatorias a estas, lo que lleva a plantear en concordancia con Gurrea y Remolina (2020) que, en definitiva, una adecuada regulación financiera debería

11. Como se citó en Urdanivia, Zambrano \& Rodríguez (2020), Fintech Radar identificó 394 Startups Fintech en México de las cuales sólo 200 empresas, que representan el 50.76\% de la industria, encajaron en la regulación. Al vencimiento del plazo para entregar solicitud ante la Comisiona Nacional Bancaria y de Valores (CNBV) para operar de manera formal, solo 85 Startups Fintech registraron su solicitud, lo que significa que del total de la industria Fintech en México solo el $21.57 \%$ podrá continuar con sus operaciones de forma legal. De las 85 empresas que registraron su solicitud, 60 solicitaron autorización para operar como instituciones de fondos de pago electrónico, y 25 como instituciones de financiamiento colectivo. basarse en tres pilares fundamentales: i) una supervisión basada en la evaluación objetiva de los riesgos asociados a cada actividad y, la manera en la que deben mitigarse los mismos, con lo cual, podrían abarcarse los nuevos riesgos asociados a la actividad propia de las fintech; ii) una regulación basada en principios que permita a las entidades cumplir con objetivos específicos y ya no con procesos concretos, atendiendo con ello la acelerada evolución del cambio tecnológico; y finalmente, iii) una regulación basa en actividades y no en instituciones (pp. 166 - 167).

A lo anterior debe sumarse que para la construcción de una iniciativa regulatoria comprensiva y sólida, que atienda el fin fundamental del Estado en materia de actividad financiera, requiere la coordinación entre los reguladores, tanto nacionales como internacionales. Asuntos como la privacidad y la protección de datos, las normas antimonopolio y de competencia, o las regulaciones de las telecomunicaciones, son cada vez más importantes para las fintechs y las instituciones financieras tradicionales en estos días, pero son, por su naturaleza, intrínsecamente interdisciplinarias y transfronterizas. Conseguir que los reguladores de un país se pongan de acuerdo puede ser casi tan difícil como lograr un consenso transfronterizo ${ }^{12}$ (Remolina, 2019).

$Y$ es que el consenso y la interdisciplinariedad entre los reguladores resultan fundamentales a la hora de eliminar los desincentivos, que hoy en día existen para el desarrollo de las fintech en algunos de los segmentos de mayor importancia para la promoción de una mayor inclusión financiera de personas naturales, trabajadores independientes, pequeñas y medianas empresas (PYMES) y emprendimientos en general, como son el de crédito digital y el de pagos digitales.

De acuerdo con el presidente de Colombia Fintech (Rincón, 2021b), en el primer caso, es necesario desarrollar a nivel normativo la

12. Traducción libre del inglés: “(..) All the while, even in a competitive world, building a coherent and robust regulatory policy requires coordination amongst regulators, both domestic and international. Matters such as data privacy and data protection, antitrust/competition, or telecommunications regulations, are becoming more important for fintechs and traditional financial institutions these days, but are by their nature inherently interdisciplinary and cross-border. Getting regulators within a country on the same page can be nearly as difficult as corralling cross-border consensus". 
categoría jurídica de crédito digital que permita dotar de seguridad jurídica y operacional al sistema de cobros. En el segundo caso, es necesario eliminar las asimetrías regulatorias que está generando el régimen tributario actual en el sistema de pagos digitales debido a que se cuenta con un régimen de retención de impuestos que genera un costo para los comercios que impacta directamente su flujo de caja, el impuesto sobre las transacciones electrónicas (4 x 1000), se genera la falta de beneficios tributarios, los riesgos de sanciones, el alto nivel de informalidad y demás temores no contribuyen al flujo de pagos digitales (Rincón, 2021a). Lo anterior, sumado a la falta de una infraestructura que fomente la interoperabilidad. El desarrollo regulatorio descrito, leyes, decretos y circulares externas, no parece tener una perspectiva que incluya en su totalidad los aspectos mencionados.

En el caso de Colombia, la expedición del decreto es muy reciente como para anticipar sus efectos y por el momento los beneficios con respecto a la implementación del sandbox regulatorio no son claros. En este contexto, expertos en el tema sugieren (Seminario, 2021) que un camino a seguir sería que las startups pasen primero por el $H u b$ de la Superintendencia, a fin de que ellos decidan a dónde recurrir, si a la Arenera del supervisor, o al sandbox de la licencia temporal establecido en el Decreto 1234 de 2020. Al respecto vale la pena mencionar que según Gurrea y Remolina (2020), la implementación de un sandbox puede resultar una estrategia más costosa e implicar por parte del supervisor un esfuerzo superior de acompañamiento, que exigirá de una mayor inversión para formar y emplear a un determinado número de personas. Lo anterior, unido a la necesidad de un alto grado de sofisticación e independencia por parte del supervisor (p. 161).

Lo que parece claro en este momento es que los sandboxes no son una solución per se, sino que más bien deben formar parte de un paquete de políticas que permitan el desarrollo prudencial de Fintech. Este paquete debe adaptarse al modelo de negocio y a los riesgos de este sector, que, en general, son diferentes a los que plantean las instituciones financieras tradicionales (Herrera \& Badillo, BID), 2018).

\section{CONCLUSIONES}

De las reflexiones realizadas a lo largo del presente documento, se puede concluir que el uso de las tecnologías continúa generando grandes oportunidades en el sector financiero a favor de los consumidores. Las mejoras en la oferta de productos y servicios, así como el aumento en el acceso y uso de estos favorecen la inclusión financiera cuyas cifras se han visto mejoradas. Sin embargo, la velocidad con la que se presentan y evolucionan los desarrollos tecnológicos plantean retos desde el punto de vista regulatorio y de supervisión que aún no pueden ser abordados al mismo ritmo. Al respecto, el Gobierno Nacional ha realizado avances considerables en materia de gestión de riesgos, manejo de la información, protección al consumidor, gestión de nuevos modelos de negocio y avances tecnológicos, los cuales parecen abonar el camino de una regulación comprensiva, pero no son suficientemente comprensivos.

El establecimiento de normas claras dependerá, en todo caso, de la colaboración entre las autoridades, las entidades del sector financiero tradicional y los emprendedores fintech. Es ese dialogo el que permitirá ajustar el marco regulatorio a las nuevas dinámicas del mercado y promover la innovación y el desarrollo tecnológico del sector financiero, de manera segura y sostenida. Se requiere generar incentivos a través del establecimiento de responsabilidades y cargas apropiadas para integrar a los nuevos actores en la dinámica del sector.

En lo que respecta al modelo de regulación adoptado por el Gobierno Nacional a través de la reglamentación de los sandboxes, es conveniente tener en cuenta la experiencia de otros países, como es el caso de México, en donde ya se evidencian las limitaciones relacionadas con la transición, desde el entorno aislado al mercado, que puede representar un shock para las startups con un core operativo limitado para tramitar licencias y permisos ante el regulador. Lo anterior, para efectos de anticipar posibles desincentivos que frenen o impidan el adecuado desarrollo no solo de los sandboxes, sino de la innovación en sí misma. 


\section{BIBLIOGRAFÍA}

- ANIF. (2018). Los servicios financieros digitales en América Latina. Disponible en http://felaban.s3-website-us-west-2. amazonaws.com/documentos_interes/ archivo20181204163600PM.pdf.

- Arner, D., Barberis , J., \& Buckley, R. (2016). "The evolution of Fintech: a new postcrisis paradigm?" Georgetown Journal of International Law. No. 47, pp. 1271-1320.

- Asobancaria. (30 de septiembre, 2019). "Gestión de riesgos en el marco de la era digital”. Semana Económica. No. 1204. Disponible en https://www.asobancaria. com/wp-content/uploads/semanaeconomica-edicion-1204.pdf

- Asobancaria. (6 de abril, 2015). "Reglamentación prudencial de las SEDPE: clave para la estabilidad del sistema y para un verdadero impulso a los procesos de inclusión financiera". Semana Económica. No. 989. Disponible en https://www. asobancaria.com/semanseconomicas / Sem_989.pdf

- Asociación Colombia Fintech. (8 de septiembre, 2020). Fintech en el Plan Nacional de Desarrollo de Colombia. Disponible en https://www. colombiafintech.co/novedades/fintechen-el-plan-nacional-de-desarrollo-decolombia

- Asociación Colombia Fintech. $(25$ de septiembre, 2018). "Fintech: la inesperada revolución que encara el sector financiero". Revista Semana. Disponible en https:// www.semana.com/economia/articulo/ reportaje-explica-las-fintech-en-colombiay-america-latina/262418/

- Banca de las Oportunidades. (2020). Informe de Inclusión financiera 2020. Bogotá: Punto Aparte Editores. Disponible en https://bancadelasoportunidades. gov.co/es/articulos/sitio/reporte-deinclusion-primer-semestre-2020
- Banca delas Oportunidades. (junio de 2019). Banca de las oportunidades. Disponible en http://www.bancadelasoportunidades. gov.co

- BBVA. (20 de noviembre, 2017). BBVA. Disponible en http://www.bbva.com

- BID. (2017). Fintech: innovaciones que no sabías que eran de América Latina $y$ el Caribe. Washington. Disponible en https:// publications.iadb.org/ bitstream/handle/11319/8265/ FINTECHInnovaciones-que-no-sabiasque-eran-de-America-Latina-y-Caribe. pdf? sequence $=2 \&$ isAllowed $=y$

- BID, BID Invest y Finnovista. (2018). Fintech América Latina 2018. Crecimiento y consolidación. Disponible en https://www. finnovista.com/informe/fintech-americalatina-2018-crecimiento-consolidacion/

- Brigard Urrutia. (2020). Reglamentada la factura electrónica como título valor. Disponible en https://bu.com.co/es/ noticias/reglamentada-la-facturaelectronica-como-titulo-valor

- Caparroso, J. (2018). "Fintech: la inesperada revolución que encara el sector financiero". Revista Semana. Disponible en https:// www.semana.com/economia/articulo/ reportaje-explica-las-fintech-en-colombiay-america-latina/262418/

- Carmona, J., \& Asociación Colombia Fintech. (2020). Informe Sectorial Fintank. Disponible en https://www. colombiafintech.co/publicaciones / informe-sectorial-fintank-2020

- Colombia Fintech. (8 de Septiembre, 2020). Colombia Fintech. Disponible en https:// www.colombiafintech.co/novedades / superfinanciera-lanza-sandbox-para-eldesarrollo-de-fintech

- DataIFX. (8 de septiembre, 2020). ¿Cómo va Colombia Fintech? Disponible en https:// www.colombiafintech.co/novedades/ 
la-regulacion-colombiana-le-da-la-espaldaa-los-criptoactivos-presidente-colombiafintech

- Finnovista. (2020). El número de startups Fintech creció un $26 \%$ en un año en Colombia, hasta las 200. Disponible en https://www.finnovista.com/wp-content/ uploads / $2020 / 05 /$ Fintech-RadarColombia.pdf

- Gurrea M., A. R. (2020). Fintech, Regtech $y$ Legaltech: fundamentos y desafíos regulatorios. Valencia: Tirant lo blanch.

- Herrera, D., \& Badillo, S. (2018). Sandbox Regulatorio en América Latina y el Caribe para el Ecosistema Fintech y el Sistema Financiero. BID. Disponible en https://publications.iadb.org/es/ publicacion/17483/sandbox-regulatorioen-america-latina-el-caribe-para-elecosistema-fintech-y-el

- Jaramillo , G. (27 de septiembre, 2020). Decreto 1235 de 2020: "Crowdfunding 2.0". (C. Fintech, Ed.) Disponible en https:// www.colombiafintech.co/novedades/ decreto-1235-de-2020-crowdfunding-2-0

- Latam Fintech Hub. (2021). Fintech in Latin America. The state of ecosystem: Funding update 2021 Q1. Disponible en https:// img.lalr.co/cms/2021/04/20155301/607 e86f93882b8934c4b4dcf_Latam-FintechFunding-2021-Q1.pdf

- Lega, F. (20 de Diciembre, 2020). Portafolio. Disponible en http://www.portafolio.co

- Magnuson, W. (2018). "Regulating Fintech". Vaderbilt Law Review, No. 71, pp. 1167 - 1226.

- Mouthon, L. (8 de Septiembre, 2020). Colombia Fintech. Disponible en https:// www.colombiafintech.co/novedades/ lo-que-debe-saber-sobre-el-ecosistemafintech-y-cinco-megatendencias-queestan-transformando-el-sector-financieroen-colombia

- Pérez D., V. (21 de diciembre, 2020). "Aprueban nueva regulación para hacer pagos electrónicos en el territorio nacional". La República. Disponible en https://www. larepublica.co/finanzas/gobierno-aprobonueva-regulacion-de-pagos-electronicosen-el-territorio-nacional-3104611

- Raventós N., J. (2020). Empresas Fintech en Colombia: sus retos y logros. (J. Raventós , \& Portafolio, Edits.) Disponible en https:// global-uploads.webflow.com/58c5b874 8712539d1de79645/5f4c796b925527 3e9bb1e437_Libro\%20EMPRESAS\%20 FINTECH\%20EN\%20COLOMBIA.pdf

- Remolina, N. (2019). Contextualizing Regulatory Sandboxes in Latin America. (F.Policy, Ed.) Disponible en https:// fintechpolicy.org/2019/01/20/ contextualizing-regulatory-sandboxes-inlatin-america/

- Restrepo E., S. (junio de 2020). El rol de las FinTech en la Inclusión Financiera: una aproximación a los servicios bancarios y el mercado crediticio colombiano. Disponible en https://www.colombiafintech.co/ publicaciones/el-rol-de-las-fintech-en-lainclusion-financiera-una-aproximaciona-los-servicios-bancarios-y-el-mercadocrediticio-colombiano

- Rincón, E. (8 de septiembre, 2020). Colombia Fintech. Disponible en http:// www.colombiafintech.co

- Rincón, E. (2021a). El desarrollo jurídico de las Fintech: Las bases regulatorias de la tecnología financiera. Bogotá: Tirant lo Blanch.

- Rincón, E. (2021b). "El ecosistema Fintech colombiano". La República, Especial: La revolución de las Fintech. Bogotá.

- Rojas, M. (17 de septiembre, 2020). Sandbox paracriptoactivosenColombia:transacciones de cash-in y cash-out. Disponible en https:// www.colombiafintech.co/novedades/ sandbox-para-criptoactivos-en-colombiatransacciones-de-cash-in-y-cash-out

- Seminario, F. (29 de marzo, 2021). Sandbox regulatorio en LatAm: los entornos de 
pruebas fintech toman forma. Disponible en https://iupana.com/2021/03/29/ sandbox-regulatorio-latam/

- Superintendencia Financiera de Colombia. (2018). "Hacia la innovación sostenible y responsable en el sector financiero". 9 Congreso de Acceso a Servicios Financieros y Medios de Pago. Cali. Disponible en https:// www.asobancaria.com/wp-content/ uploads/0.-Jorge-Casta\%C3\%B1o-Superintendente-Presentaci\%C3\%B3n-InnovaCali-abril-2018.pdf

- Superintendencia Financiera de Colombia. (2020). Avances fintech y el Sandbox regulatorio. Cali.

- Superintendencia Financiera de Colombia. (mayo de 2020). Manual de Funcionamiento de la Arenera (Versión 2.0). Disponible en https://www.superfinanciera.gov.co/ descargas/institucional/pubFile1030976/ manualarenera.pdf

- Urdanivia G., E., Zambrano R., A., \& Rodríguez G., T. (2020). "El impacto de la
Ley Fintech a la Industria de la Tecnología Financiera y el reto de establecer un Gobierno Corporativo". Boletín Científico de las Ciencias Económico Administrativas del ICEA, pp. 21-29.

- US National Ecnomic Council. (2017). Disponible en https://obamawhitehouse. archives.gov/sites/obamawhitehouse. archives.gov/files/documents / A $\% 20$ Framework\%2 0 for $\% 20$ Fin Tech $\% 20$ _ FINAL.pdf

- Vargas, P. A. (29 de enero, 2021). “En el sandbox, habrá nueve alianzas que podrán realizar pruebas con criptoactivos". La República. Disponible en https:// www.larepublica.co/finanzas/estasson-las-alianzas-que-haran-pruebascon-criptoactivos-en-el-sandbox-de-lasuperfinanciera-3117891

- Zapata, E. (8 de Septiembre, 2020). Colombia Fintech. Disponible en https://www. colombiafintech.co/novedades/estado-delmovimiento-fintech-en-colombia 